\title{
Long-Lasting Induction of Astrocytic Basic Fibroblast Growth Factor by Repeated Injections of Amphetamine: Blockade by Concurrent Treatment with a Glutamate Antagonist
}

\author{
Cecilia Flores, Demetra Rodaros, and Jane Stewart \\ Center for Studies in Behavioral Neurobiology, Department of Psychology, Concordia University, Montreal, Quebec, \\ Canada, H3G 1 M8
}

Repeated administration of stimulant drugs leads to lasting changes in their behavioral and neurochemical effects. These changes are initiated by drug actions in the somatodendritic regions of midbrain dopaminergic neurons in the ventral tegmental area (VTA) and substantia nigra pars compacta (SNc) and continue to develop for a period of time after termination of drug treatment. Here we show that repeated administration of amphetamine $(3.0 \mathrm{mg} / \mathrm{kg}$, i.p.; three injections, once every other day) results in sustained increases in basic fibroblast growth factor immunoreactivity (bFGF-IR) in both VTA and SNc, 200$500 \%$ over that seen in saline-treated animals. Increases were observed $24 \mathrm{hr}, 72 \mathrm{hr}, 1$ week and 1 month after the last drug injection. Because glutamate participates in the development of sensitization to stimulant drugs, we assessed the effect of the glutamate antagonist, kynurenic acid (KYN), on amphetamineinduced bFGF-IR. Coadministration of KYN prevented the in- creases in bFGF-IR in both VTA and SNc assessed 1 week after the amphetamine treatment. No changes in bFGF-IR were observed in the nucleus accumbens or dorsal striatum. bFGF-IR was found to be associated with astrocytes and not with dopaminergic neurons. These findings suggest that sustained enhancement of astrocytic bFGF expression in DA somatodendritic regions is a mechanism whereby stimulant drugs exert enduring effects on midbrain DA function. We hypothesize that increased glutamatergic activity elicited by amphetamine and other stimulant drugs places excessive demands on the functioning of DA neurons recruiting regulatory and neuroprotective processes that lead to enduring changes in DA neuron functioning and connectivity.

Key words: bFGF; amphetamine; sensitization; glutamate; dopamine; neurotrophic factors
Repeated administration of stimulant drugs, such as amphetamine, cocaine, and morphine, leads to an enduring enhancement of their behavioral activating effects that is associated with changes in the functioning of the midbrain dopamine (DA) system (Robinson and Becker, 1986; Kalivas and Stewart, 1991). One frequently reported change is enhanced DA overflow in striatal regions in response to acute drug challenge. Importantly, this change is long-lasting (Robinson et al., 1988; Paulson et al., 1991; Robinson, 1991; Kalivas and Duffy, 1993; Wolf et al., 1993; Paulson and Robinson, 1995; Heidbreder et al., 1996) and takes time to develop, usually not observed until 2-3 weeks after the last drug injection (Kolta et al., 1985; Vezina, 1993; Wolf et al., 1993; Paulson and Robinson, 1995).

The events that lead to enhanced DA functioning are initiated by the actions of these drugs in the somatodendritic region. Repeated administration of amphetamine into the ventral tegmental area (VTA), but not into the nucleus accumbens (NAcc), is sufficient to induce behavioral sensitization and increased DA release in striatal regions in response to systemic injections of stimulant drugs and to intra-NAcc amphetamine (Kalivas and Weber, 1988; Vezina and Stewart, 1990; Hooks et al., 1992;

Received July 28, 1998; revised Aug. 28, 1998; accepted Sept. 3, 1998.

This work was supported by grants from the Medical Research Council of Canada and Fonds pour la Formation de Chercheurs et l'Aide à la Recherche (FCAR, Quebec). C.F. was supported by a graduate fellowship from Concordia University. We thank Sean Cain for technical assistance.

Correspondence should be addressed to Dr. Jane Stewart, Center for Studies in Behavioral Neurobiology, Department of Psychology, Concordia University, 1455 de Maisonneuve Boulevard, Montreal, Quebec, Canada, H3G 1M8.

Copyright (C) 1998 Society for Neuroscience $\quad 0270-6474 / 98 / 189547-09 \$ 05.00 / 0$
Vezina, 1993; Perugini and Vezina, 1994; Cador et al., 1995; Bjijou et al., 1996). Furthermore, studies have shown that glutamate plays an important role in the initiation of the changes accompanying sensitized responding. Coadministration of NMDA or AMPA receptor antagonists, given systemically or directly into the VTA, can prevent the development of stimulantinduced behavioral sensitization (Karler et al., 1989, 1990, 1991, 1994; Wolf and Khansa, 1991; Kalivas and Alesdatter, 1993; Stewart and Druhan, 1993; Wolf and Jeziorski, 1993; Cador et al., 1997; Li et al., 1997) and the increases in DA in striatal regions (Jake-Matthews et al., 1997). NMDA antagonists also prevent alternations in the VTA seen early after drug treatment (Wolf et al., 1994; Masserano et al., 1996).

There is now evidence that extracellular glutamate is increased in the VTA during (Kalivas and Duffy, 1995a, 1998) or after (Xue et al., 1996; Wolf and Xue, 1998) injections of amphetamine or D1 DA receptor agonists. Repeated administration of stimulants leads to a transient increase in the responsiveness of DA neurons to glutamate (White et al., 1995) mediated by AMPA receptors (Zhang et al., 1997). GluR1 AMPA subunit immunoreactivity (IR) is increased in the VTA soon after termination of cocaine injections (Fitzgerald et al., 1996), and a similar trend is suggested after amphetamine (Wolf, 1998). Interestingly, viralmediated gene transfer upregulation of GluR1 in the VTA has been shown to induce a sensitized-like response to morphine (Carlezon et al., 1997).

Drug-induced glutamatergic activity in somatodendritic regions may place excessive demands on the functioning of DA neurons. In fact, injury-like changes, such as increased glial fibril- 
lary acidic protein (GFAP)-IR, decreased neurofilament protein, axonal transport (Self and Nestler, 1995), and DA neuronal size (Sklair-Tavron et al., 1996) have been reported after exposure to stimulant drugs. We postulated, therefore, that the long-term changes induced in DA neurons by stimulant drugs might result from the actions of neuroprotective factors, such as basic fibroblast growth factor (bFGF), normally recruited in response to injury. bFGF promotes growth and survival of midbrain DA cells (Chadi et al., 1993; Bouvier and Mytilineou, 1995; Takayama et al., 1995; Hou et al., 1997); sustained increases in bFGF-IR have been observed in the VTA and substantia nigra compacta $(\mathrm{SNc})$, but not in terminal regions, after 6-hydroxydopamine (6-OHDA) lesions (Chadi et al., 1994). Interestingly, glutamate increases bFGF-IR in astrocytes (Pechan et al., 1993), and bFGF upregulates GluR1 expression in cultured hippocampal neurons (Cheng et al., 1995).

We examined, therefore, changes in bFGF-IR in midbrain DA regions at different time intervals after repeated amphetamine treatment and investigated a role for glutamate by examining bFGF-IR after concurrent treatment with the nonselective ionotropic glutamate receptor antagonist kynurenic acid.

\section{MATERIALS AND METHODS}

Subjects. Male Wistar rats weighing 300-350 gm at the beginning of the experiment served as subjects. Rats were housed individually in standard stainless steel hanging boxes with ad libitum access to tap water and rat chow, and were maintained on a $12 \mathrm{hr}$ light/dark cycle.

Drugs and antibodies. D-amphetamine sulfate (AMPH) was obtained from SmithKline Beecham Pharma (Oakville, Ontario) and was dissolved in physiological saline. Kynurenic acid (KYN, 4hydroxyquinoline-2-carboxylic acid) was obtained from Sigma (St. Louis, $\mathrm{MO}$ ) and was suspended in distilled water containing $2 \%$ Tween 80 (vehicle solution). bFGF immunoreactivity was detected using a mouse monoclonal antibody purchased from Upstate Biotechnology (Lake Placid, NY). This antibody recognizes the biologically active isoform of bFGF (Matsuzaki et al., 1989). For GFAP and tyrosine hydroxylase (TH) immunoreactivity, a mouse monoclonal antibody obtained from Sigma and a rabbit polyclonal antibody obtained from Eugene Tech (Ramsay, NJ) were used, respectively.

Immunohistochemistry. Animals received an overdose of sodium pentobarbital $(120 \mathrm{mg} / \mathrm{kg})$ and were perfused transcardially with $200 \mathrm{ml}$ of ice-cold PBS followed by $100 \mathrm{ml}$ of an ice-cold solution of $4 \%$ paraformaldehyde (w/v) and $15 \%$ picric acid (v/v) in $0.1 \mathrm{M}$ phosphate buffer (PB, $\mathrm{pH}$ 6.9). When the perfusion was completed, the brains were removed and placed overnight in the fixative solution at $4^{\circ} \mathrm{C}$. Coronal sections, $50-\mu \mathrm{m}$-thick, were cut on a vibratome and stored overnight in $\mathrm{PB}$ at $4^{\circ} \mathrm{C}$. bFGF immunohistochemistry was then detected according to the ABC method (Hsu et al., 1981). Briefly, free-floating tissue sections were incubated for $24 \mathrm{hr}$ at $4^{\circ} \mathrm{C}$ with the anti-bFGF antibody diluted to 1:500 with $0.3 \%$ Triton X-100 (Sigma) in PB and 1\% normal horse serum (Vector Laboratories, Burlingame, CA). After incubation in the primary antibody, sections were rinsed three times in cold $\mathrm{PB}$ and incubated for $1 \mathrm{hr}$ at room temperature (RT) in a solution of rat adsorbed biotinylated anti-mouse antibody (Vector) diluted 1:200 with PB and 1\% normal horse serum. After three 5 min washes in cold PB, sections were then incubated in an avidin-horseradish peroxidase complex (Vectastain Elite $\mathrm{ABC}$ Kit, Vector) for $30 \mathrm{~min}$ at RT, and rinsed again three times $(5 \mathrm{~min}$ each) in cold PB. Sections were then incubated for $10 \mathrm{~min}$ at RT and under constant agitation in a solution of $0.05 \% 3,3^{\prime}$-diaminobenzidine (DAB, Sigma) in PB. Without washing, the sections were then transferred to a DAB-PB solution, $\mathrm{pH} 7.8$, containing $0.01 \% \mathrm{H}_{2} \mathrm{O}_{2}$, which catalyzed the reaction, and $8 \% \mathrm{NiCl}_{2}$, which darkened the reaction product. Sections were incubated in this solution at RT and under constant agitation for $8 \mathrm{~min}$. Special care was taken to maintain this time rigorously constant for all sections processed within one single experiment and throughout the entire study. Three $10 \mathrm{~min}$ washes with cold PB terminated this final incubation.

Double-labeling for bFGF-GFAP and for bFGF-TH was performed by processing the sections; first, for bFGF immunohistochemistry and then for either GFAP or TH immunohistochemistry. GFAP and TH immunolabeling was performed by using the $\mathrm{ABC}$ method. The antiGFAP antibody was used at a concentration of 1:500 and the anti-TH at a concentration of 1:2000. For TH immunohistochemistry, sections were preincubated in $0.3 \%$ Triton X-100 PB and $1 \%$ normal goat serum for 1 $\mathrm{hr}$ at RT. For both GFAP and TH immunohistochemistry no $\mathrm{NiCl}_{2}$ was added to the DAB-PB- $\mathrm{H}_{2} \mathrm{O}_{2}$ solution to obtain a lighter reaction product.

Histology. Processed sections were wet-mounted onto gelatin-coated slides and were allowed to dry for at least $1 \mathrm{~d}$ before being hydrated in distilled water and gradually dehydrated throughout a series of graded alcohol solutions. Midbrain sections processed for bFGF-IR were lightly counterstained with $0.1 \%$ cresyl violet to demonstrate anatomical landmarks. Slides were cleared in xylene and coverslipped with Permount.

Image analysis. Immunostained sections were examined under a Leica microscope (Leitz DMRB). For quantitative analysis of bFGF-IR, images of sampling areas of the VTA, SNc, NAcc shell, NAcc core, and dorsal region of the striatum (STR) were digitized using a computerized image-analysis system (NIH Image 1.6). Structure boundaries were defined according to the Paxinos and Watson (1997) stereotaxic atlas. Sampling areas of VTA and SNc were taken from sections corresponding to plates 38 and 39, and sampling areas of NAcc shell, NAcc core, and STR were taken from sections corresponding to plates 11, 12, and 13.

For each brain, three images from each structure, taken from three different sections, were digitized and were assigned code names. The number of bFGF-positive cells in each image was then counted by two individuals who were blind to the code assignment. The means of the cell counts from the three sections for each structure in each animal were calculated by each observer. Correlation coefficients were calculated between the mean cell counts obtained by each observer. When these correlation coefficients were $\geq 90 \%$, the cell counts obtained by the two observers were averaged; the resulting values were used to calculate the group means \pm SEM per area. Importantly, brains of subjects from each of the experimental conditions were always processed in parallel from perfusion to image analysis.

\section{DESIGN AND PROCEDURES}

Figure 1 outlines the timing of the treatment and experimental manipulations in experiments 1 and 2 .

\section{Experiment 1: Time course}

This experiment was conducted to determine levels of bFGF-IR in midbrain dopaminergic regions (VTA and $\mathrm{SNc}$ ) at several time points after repeated administration of AMPH. In the preexposure phase, different groups of rats were given injections of either AMPH (3 mg/kg, i.p.) or saline (SAL) in the morning (10:00 A.M.) on days 1,3 , and 5 , in the colony room. This or similar treatment regimens are known to produce behavioral sensitization, and we have confirmed this under the conditions used in the present experiments (our unpublished observations). Groups of AMPH-treated $(n=5)$ and saline-treated $(n=4)$ rats were killed and perfused at each time period, $24 \mathrm{hr}, 72 \mathrm{hr}, 1$ week, and 1 month after the last injection, and their brains were processed for bFGF immunohistochemistry.

\section{Experiment 2: Role of glutamate}

Because glutamate plays a role in the development of sensitization, we investigated whether a glutamate antagonist would prevent the effect of AMPH on bFGF-IR. Previous studies have shown that both NMDA and AMPA receptor antagonists prevent the development of behavioral sensitization as well as some of its cellular correlates (Wolf, 1998). We tested, therefore, whether the nonselective ionotropic glutamate receptor antagonist, kynurenic acid (KYN) (Stone, 1993; see also Taber and Fibiger, 1995), would prevent the AMPH-induced increases in bFGF-IR observed in the VTA and SNc.

Different groups of rats undergoing the same AMPH or saline regimen as in experiment 1 on days 1,3 , and 5, were treated twice daily, on days 1-10, with the nonselective ionotropic glutamate receptor antagonist KYN (100 mg/kg, i.p. at 9:00 A.M. and 6:00 


\section{EXPERIMENT 1}

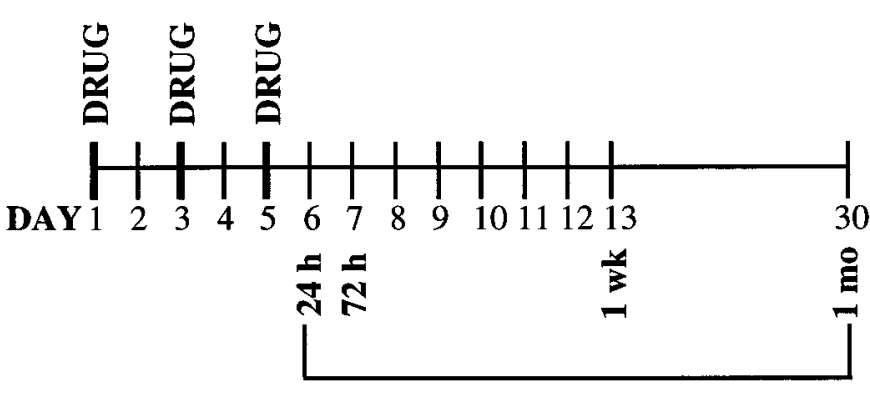

PERFUSION

\section{EXPERIMENT 2}

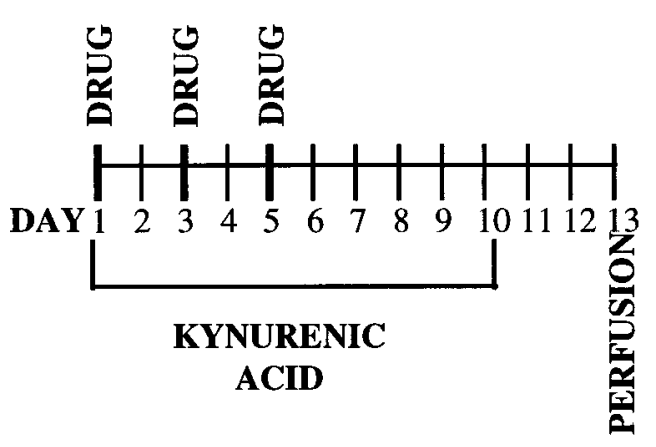

Figure 1. Diagram outlining the timing of the treatment and experimental manipulations in experiments 1 and 2 .

P.M.) or with vehicle (either $2 \%$ Tween 80 or saline). Because there is evidence that even a single injection of amphetamine can induce an increase in extracellular glutamate (Xue et al., 1996), and because, therefore, the processes involved in bFGF expression might be initiated after each amphetamine injection, we tried to ensure that glutamate receptors were blocked not only while amphetamine was acting, but also in the period between injections. In addition, we decided to continue the treatment for a few days after AMPH termination because we had found in experiment 1 that the expression of bFGF increased over time after the last injection of amphetamine. Because surgical penetration of the brain would be likely to induce bFGF expression in itself, we chose to administer the glutamate receptor antagonist systemically. Animals from each of the four groups: vehicle-AMPH (VTA, $n=8$; SNc, $n=7)$, vehicle-saline $(n=8)$, KYN-AMPH $(n=4)$, and $\mathrm{KYN}$-saline $(n=4)$ were killed and perfused 1 week after the last AMPH or saline injection (72 hr after the last KYN or vehicle injection), and their brains were processed for bFGF-IR. This time point was chosen on the basis of the substantial increase in bFGF-IR observed in experiment 1 in both VTA and SNc. Brain sections from both DA cell body regions (VTA and SNc) and DA terminal regions (NAcc shell and core and STR) were analyzed.

\section{Double-labeling experiment}

To determine the nature of the cells expressing bFGF in the regions analyzed, double-labeling for bFGF-GFAP and for bFGF-TH was conducted on the brains of two rats that received three injections of either AMPH or SAL in the same regimen used in experiment 1 and that were killed and perfused 1 week after the last AMPH or SAL injection.

\section{Statistical analysis}

The data were analyzed with one-way ANOVAs. Post hoc analyses of significant effects were made using Fisher's PLSD tests. The analyses were done using the number of bFGF-IR cells per square millimeter. The data in the figures are presented as percent of controls. In the time course study (experiment 1), because no differences in bFGF expression were found between the saline-treated groups at the different time periods in either the VTA or SNc, the data from all saline-treated groups were combined and compared with the AMPH-treated groups at each time interval.

\section{RESULTS}

Repeated amphetamine induces long-lasting expression of bFGF in DA somatodendritic regions

Repeated intermittent AMPH administration $(3.0 \mathrm{mg} / \mathrm{kg}$, i.p., three injections, once every other day) resulted in an increase in bFGF-IR in both VTA and SNc over that seen in saline-treated animals. As shown in Figure 2, a significant increase in bFGF-IR in the VTA was observed $24 \mathrm{hr}(286 \pm 48 \%)$ after the last AMPH injection. This increase was sustained $72 \mathrm{hr}(215 \pm 71 \%)$ and 1 week $(284 \pm 51 \%)$ after the last drug injection and was further increased 1 month $(418 \pm 25 \%)$ after treatment termination. Within the SNc, a significant increase in bFGF-IR was observed $24 \mathrm{hr}(303 \pm 83 \%)$ and $72 \mathrm{hr}(363 \pm 72 \%)$ after the last drug injection (Fig. 2). Levels of bFGF-IR within this region appeared to peak at 1 week $(542 \pm 53 \%)$ and remained elevated (434 \pm $45 \%) 1$ month after treatment termination. The maximum number of bFGF-immunoreactive cells observed within the VTA of AMPH-treated animals was three times higher than that of saline-treated animals and was observed 1 month after the last drug injection. In the case of the SNc, maximum levels of bFGFIR, observed 1 week after the last AMPH injection, were almost five times higher than those observed in saline-treated rats.

Figure 3 shows examples of bFGF-IR in the VTA and SNc of animals that were treated with either saline or AMPH and that were killed 1 week after the last injection. It can be seen that there was a greater number of darkly labeled bFGF-positive cells in AMPH-treated animals in these areas.

\section{Amphetamine-induced bFGF-IR is blocked by a glutamate receptor antagonist}

\section{$D A$ somatodendritic regions}

As can be seen in Figure 4, once again, animals treated with AMPH showed a significant increase in bFGF-IR in both the VTA and SNc compared with saline-treated animals. This difference between AMPH- and saline-treated animals was prevented by the coadministration of the glutamate receptor antagonist KYN. In neither area was there any effect on bFGF-IR of KYN alone (group KYN-saline).

It will be noted that bFGF-IR levels in the VTA and SNc in the vehicle control group were higher in experiment 2 than they were in the control group in the time course experiment (experiment 1). Thus, the percent increase in bFGF-IR induced by amphetamine in experiment 2 was smaller than the increase observed in the amphetamine group 1 week after the last injection in experiment 1 (Figs. 2, 4). It is likely that stress was the source of the difference observed in bFGF-IR levels between the two control groups. In experiment 2 , kynurenic acid was administered to rats 

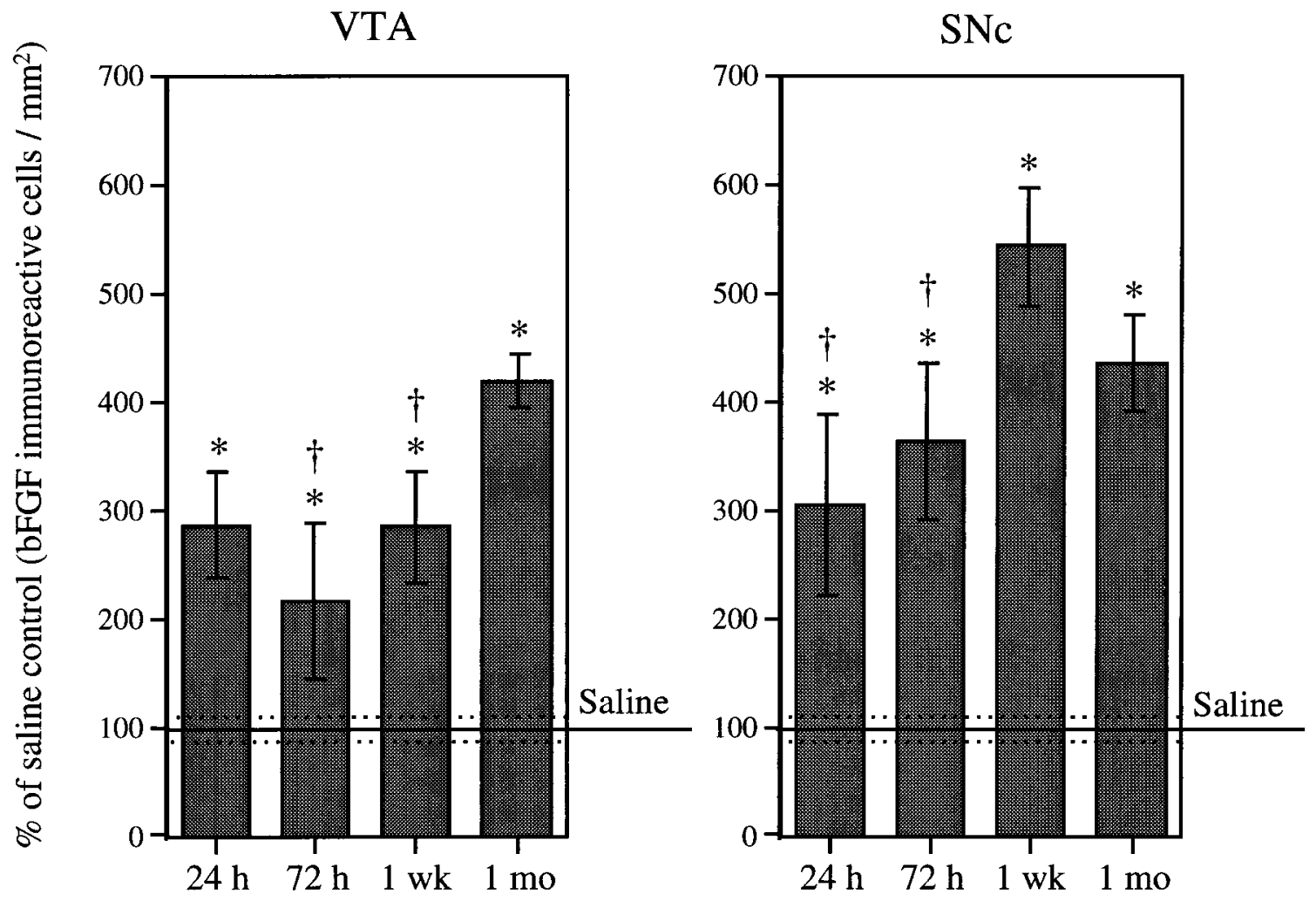

Figure 2. Time course. Mean ( \pm SEM) percent of saline control (bFGF-immunoreactive cells per square millimeter) in the VTA and SNc in groups $(n=5$ per group) of amphetamine-treated animals $(3.0 \mathrm{mg} / \mathrm{kg}$, i.p., on three occasions, once every other day) killed $24 \mathrm{hr}, 72 \mathrm{hr}, 1 \mathrm{week}$, and $1 \mathrm{month}$ after the last injection. One hundred percent (100) represents the mean (solid line) and SEM (dashed lines) counts from saline-treated animals that were killed at each of the time intervals after the last injection $(n=15)$. ANOVAs performed on the actual counts showed a significant effect of drug treatment $\left(V T A, F_{(4,30)}=11.61 ; p=0.0001 ; S N c, F_{(4,30)}=16.85 ; p<0.0001\right)$. Asterisks indicate significant differences from the saline group, $p<0.01 ;$ daggers indicate significant differences from the peak, $p<0.05$.

chronically. Whereas animals in the time course experiment were injected only three times, animals in experiment 2 were given two or three injections a day for $10 \mathrm{~d}$. Numerous studies have shown that repeated exposure to stress can lead to changes in the responsiveness of the midbrain DA system (Kalivas and Duffy, 1995b). Thus, studies of the effects of stress on bFGF-IR in midbrain DA regions appear warranted.

\section{DA terminal regions}

As shown in Figure 5, levels of bFGF-IR in the STR and in the NAcc shell were not affected by AMPH treatment. No changes in bFGF-IR within these regions were observed in either vehicle- or KYN-treated rats. Within the NAcc core, the AMPH group exhibited a small but nonsignificant increase in bFGF-IR in comparison to the saline group. This elevation was not observed in AMPH-treated rats that received $\mathrm{KYN}$ administration (Fig. 5).

\section{bFGF-IR was found in astrocytes and not in DA neurons}

In both AMPH- and saline-treated rats, double-label experiments indicated that (1) in all areas examined bFGF-positive cells were also GFAP-positive, (2) many, but not all, astrocytes expressed bFGF-IR, and (3) TH-positive cells did not exhibit bFGF-IR. These observations are illustrated in Figure 6, which shows THbFGF (Fig. 6A) and GFAP-bFGF (Fig. 6B) double-labeling in a region of the VTA of one AMPH-treated animal that was killed 1 week after the last drug injection. Similar findings were ob- served in the brain of a saline-treated animal (data not shown). These observations are in agreement with previous studies showing that the antibody used in this study detects bFGF-IR in astrocytic nuclei (Szele et al., 1995).

\section{DISCUSSION}

The primary purpose of this study was to determine whether repeated injections of amphetamine would induce increases in bFGF-IR expression in midbrain DA regions after termination of treatment. It was found that after three intermittent injections of $3.0 \mathrm{mg} / \mathrm{kg}$ of D-amphetamine sulfate administered intraperitoneally, once every other day, there were large increases in bFGF-IR levels in the somatodendritic region of the midbrain DA neurons in the VTA and SNc. These increases occurred as early as $24 \mathrm{hr}$ after the last injection, increased over a period of 1 week, and were clearly evident at 1 month. The results from the doublelabeling experiments revealed that bFGF-IR in these regions was associated with astrocytes and not with DA neurons themselves.

As discussed in the introductory remarks, enhanced DA transmission, including increased DA overflow in striatal terminal regions in response to drug or stress challenges, follows repeated administration of stimulant drugs (Robinson and Becker, 1986; Kalivas and Stewart, 1991; but see Kuczenski et al., 1997). When found, these changes take time to develop, appearing 1-3 weeks after the last drug injection (Kolta et al., 1985; Vezina, 1993; Wolf et al., 1993; Paulson and Robinson, 1995). In the present study, we postulated that the long-term changes induced in DA neurons by 


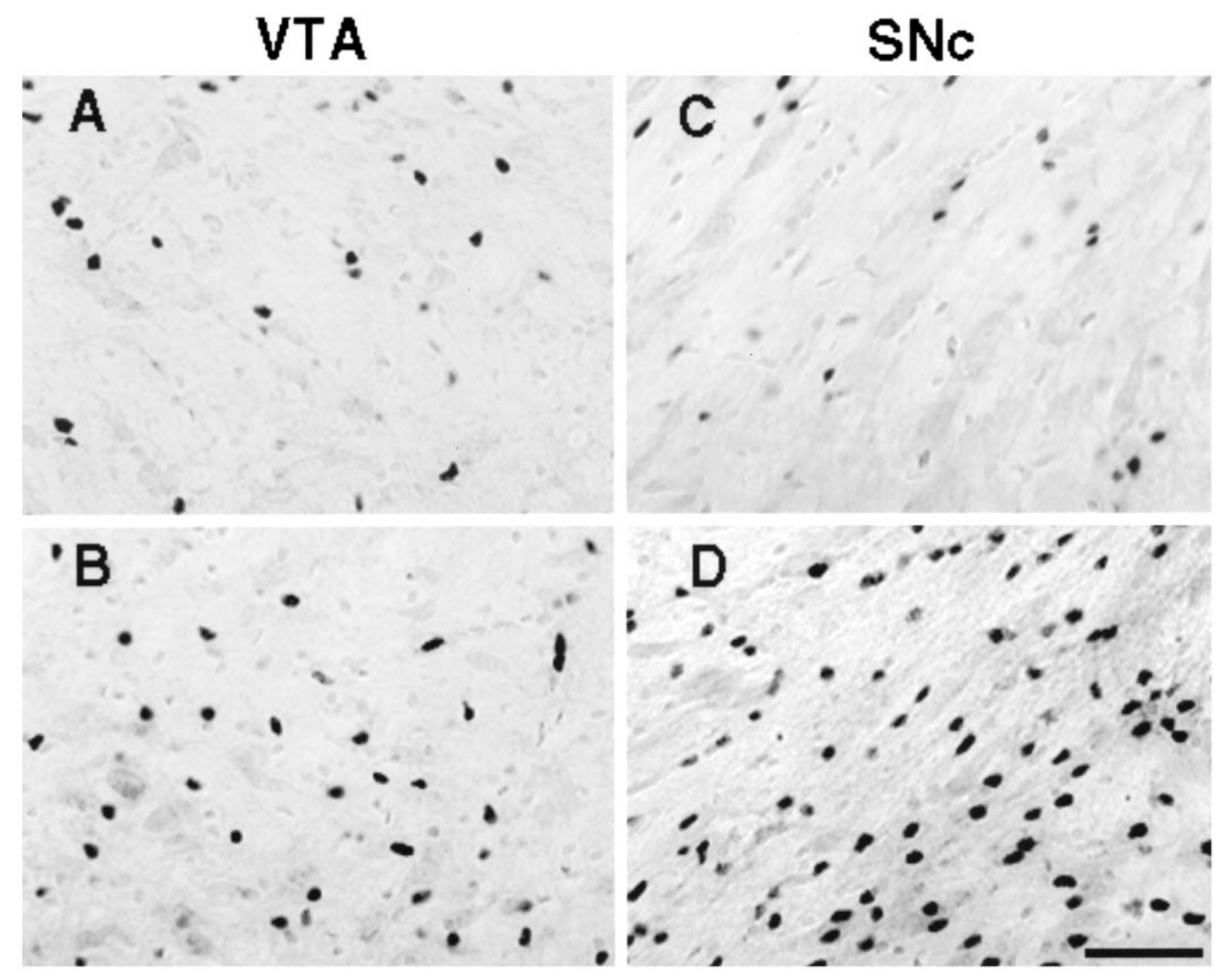

Figure 3. Digitized images showing darkly labeled bFGF-immunoreactive cells in tissue lightly counterstained with cresyl violet (revealing both glia and larger neurons). Images were taken from representative animals that were injected, intraperitoneally, with either saline or amphetamine (3.0 $\mathrm{mg} / \mathrm{kg}$ ), on three occasions, once every other day, and killed 1 week later. Images $A$ (saline) and $B$ (amphetamine) were taken from the $V T A$, and images $C$ (saline) and $D$ (amphetamine) were taken from the $S N c$. Scale bar, $50 \mu \mathrm{m}$. Cell counts included only darkly labeled profiles that were in the same plane of focus.

stimulant drugs might result from the actions of neuroprotective factors, such as bFGF, recruited in response to the effects of amphetamine in regions of the midbrain DA system. We found, in fact, that bFGF expression in DA cell body regions increased during the period that is usually needed for sensitization of the DA response to develop. Furthermore, the enhancement in bFGF-IR was persistent, as are the changes in DA function induced by stimulant drugs. These findings raise the possibility, therefore, that increased bFGF expression in the DA somatodendritic region is one of the events that leads to the development and maintenance of the neural changes underlying sensitization. Experiments using bFGF antibodies to determine whether bFGF plays a critical role in the development of sensitization to amphetamine are currently underway.

The possibility that bFGF expression is related to the development of sensitization to amphetamine is also suggested by the findings from experiment 2, showing that the glutamate receptor antagonist kynurenic acid $100 \mathrm{mg} / \mathrm{kg}$, given intraperitoneally twice a day during the period of amphetamine treatment and for
$5 \mathrm{~d}$ after termination of treatment, blocked the amphetamineinduced increase in bFGF expression in VTA and SNc seen after 1 week in animals given the vehicle. These results, suggesting that the enhancement in bFGF-IR in DA cell body regions seen after amphetamine treatment is mediated by glutamate, are supported by two additional sets of data, one showing that glutamate induces bFGF expression in astrocytes (Pechan et al., 1993) and another showing that glutamate overflow in DA cell body regions increases during (Kalivas and Duffy, 1995a, 1998) or after (Xue et al., 1996; Wolf and Xue, 1998) amphetamine administration. Glutamate is known to play a crucial role in the development of sensitization to amphetamine. There is considerable evidence that treatment with NMDA and AMPA antagonists prevents sensitization to amphetamine (for review, see Wolf, 1998). Therefore, in view of the present findings, one may hypothesize that glutamate antagonists block the development of sensitization to amphetamine by preventing bFGF increased expression. Additional experiments are clearly necessary to test this hypothesis directly and to determine more precisely which glutamate recep- 
Figure 4. Role of glutamate in DA somatodendritic regions. Mean ( \pm SEM) percent of vehicle-saline control (bFGF-immunoreactive cells per square millimeter) in the VTA and SNc in groups of animals treated daily with kynurenic acid ( $n=4$ per group) or vehicle (VTA, $n=8 ; S N c, n=7)$, given three injections of saline or amphetamine $(3.0 \mathrm{mg} /$ $\mathrm{kg}$ ), and killed 1 week after the last injection of saline or amphetamine; $100 \%$ represents the mean (solid line) and SEM (dashed lines) counts from vehicle-saline-treated animals $(n=8)$. See Materials and Methods for details. ANOVAs performed on the actual counts showed significant group difference in each area $\left(V T A, F_{(3,20)}=3.77 ; p<0.05 ; S N c\right.$, $\left.F_{(3,19)}=4.11 ; p<0.05\right)$. Asterisks indicate that the vehicle-amphetamine group was significantly different from all other groups ( $p<$ $0.05)$.
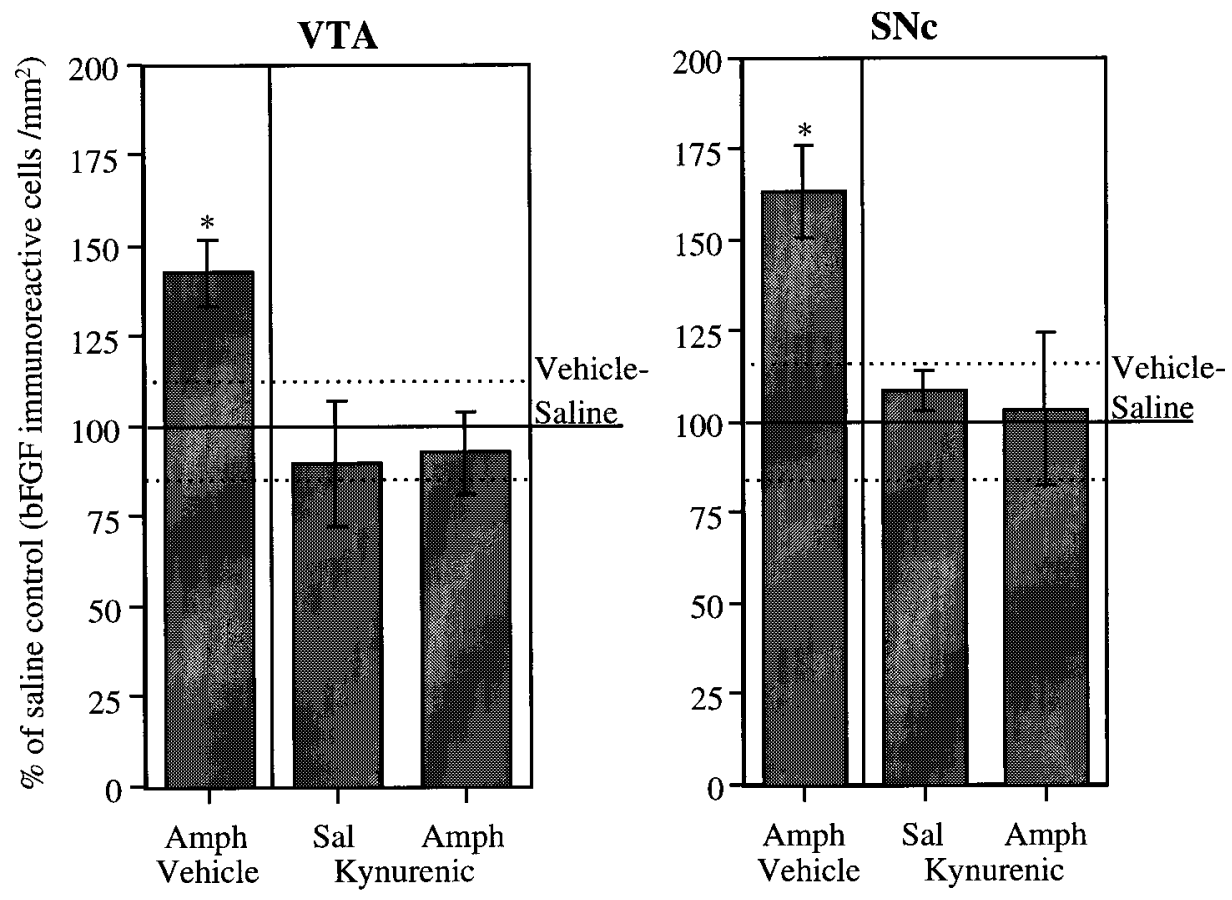

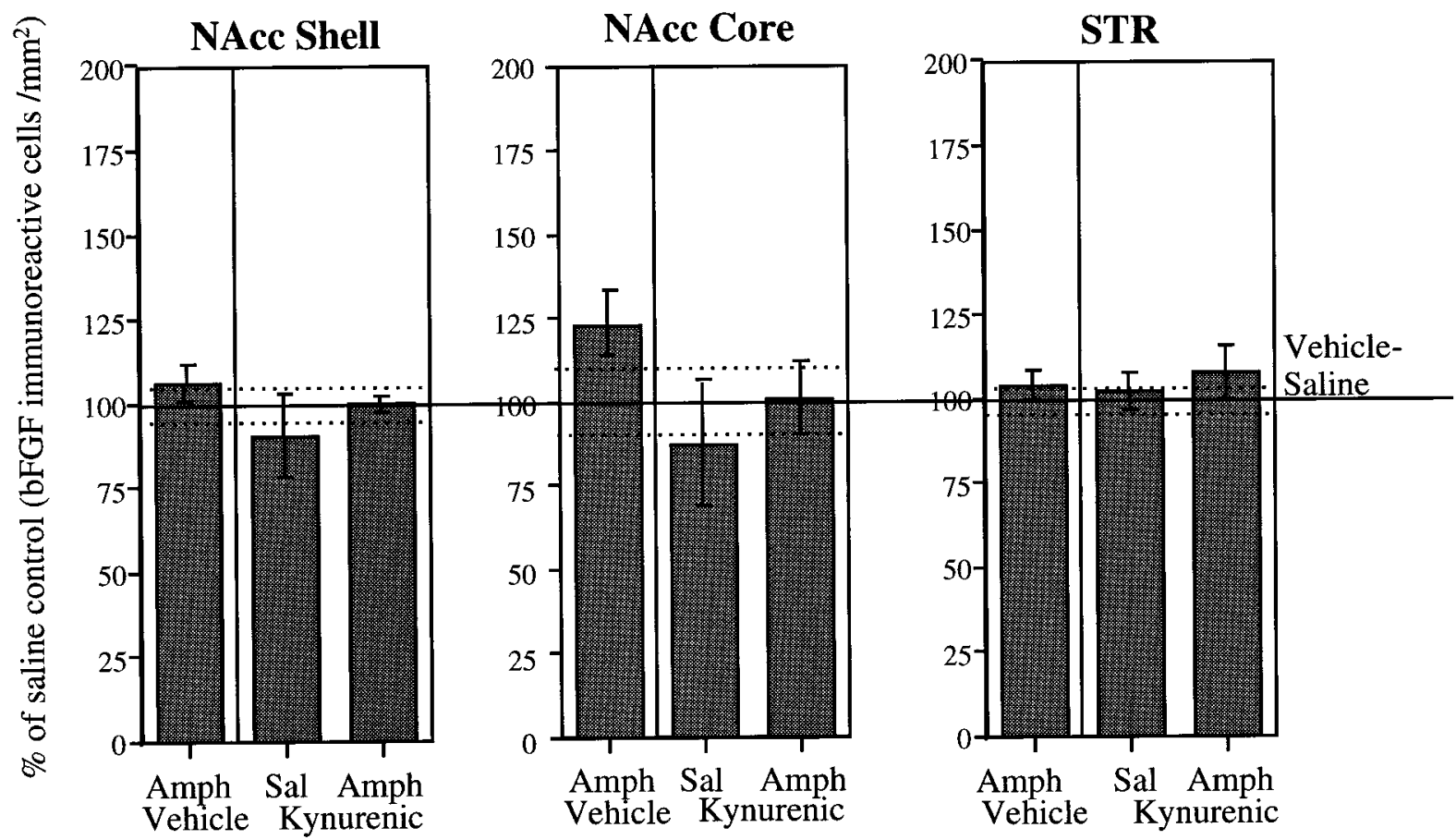

Figure 5. Role of glutamate in DA terminal regions. Mean ( \pm SEM) percent of vehicle-saline control (bFGF-immunoreactive cells per square millimeter) in the NAcc shell and core and in the dorsal region of the STR in groups ( $n=4$ per group) of animals treated daily with kynurenic acid or vehicle ( $n=8$ per group), given three injections of saline or amphetamine $(3.0 \mathrm{mg} / \mathrm{kg})$, and killed 1 week after the last injection of saline or amphetamine; $100 \%$ represents the mean (solid line) and SEM (dashed lines) counts from vehicle-saline-treated animals (NAcc Core and STR, $n=7$; NAcc Shell, $n=8)$. See Materials and Methods for details. ANOVAs performed on the actual counts showed no significant group differences in any of the areas $\left(\operatorname{NAcc}\right.$ Shell, $F_{(3,20)}=0.89 ; N A c c$ Core,$\left.F_{(3,19)}=1.52 ; \operatorname{STR}, F_{(3,19)}=0.21\right)$.

tors are involved. We have recently found that amphetamineinduced increases in bFGF-IR can be blocked by a selective NMDA receptor antagonist (our unpublished observations).

The results of the present study also revealed that the amphetamine-induced increases in bFGF-IR occurred in the somatodendritic regions of the DA neurons and not in the termi- nal regions, in the NAcc core and shell, or in the STR. As mentioned earlier, it is well established that the events that lead to sensitization are initiated by the actions of amphetamine in the DA cell body region and not in these terminal regions (Kalivas and Weber, 1988; Vezina and Stewart, 1990; Hooks et al., 1992; Cador et al., 1995). Moreover, injections of glutamate antagonists 

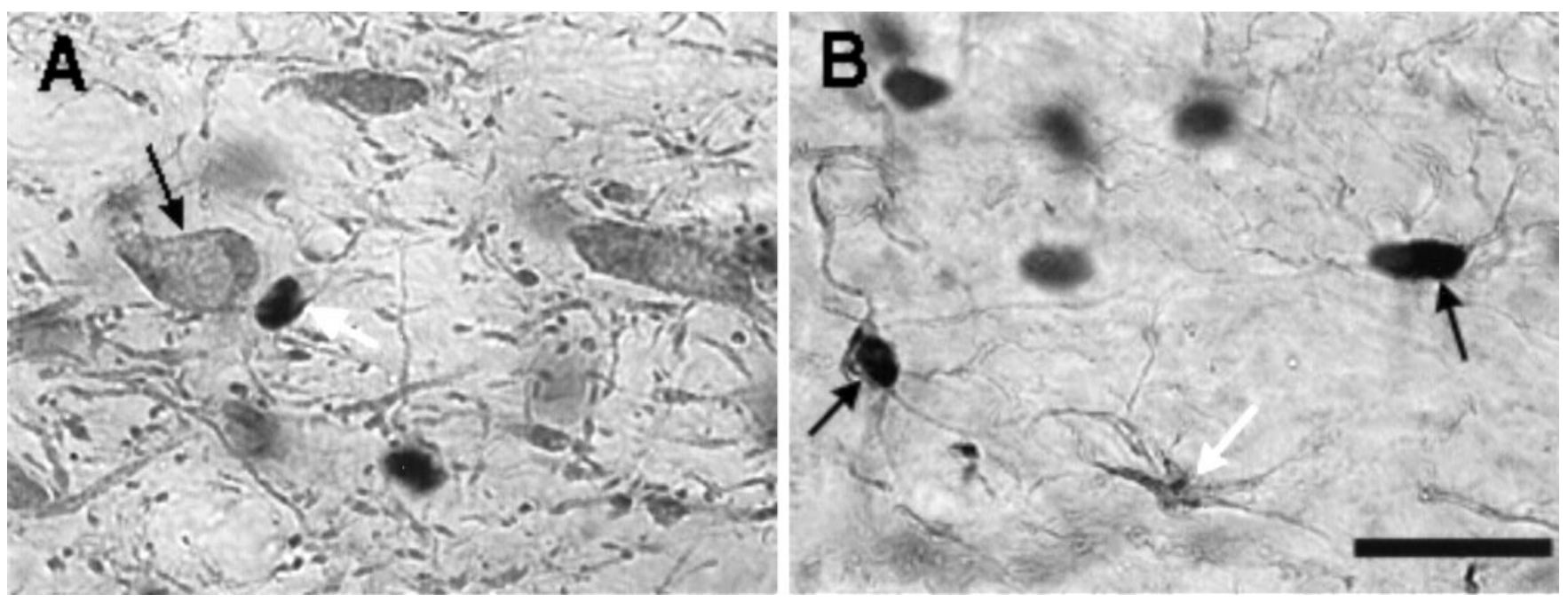

Figure 6. Digitized images of VTA sections from an animal treated with amphetamine as in experiment 1 and killed 1 week after the last injection. The large black blobs indicate bFGF immunoreactivity. $A$, Section labeled with both TH and bFGF. bFGF-IR (indicated by the white arrow) was not found within the large TH-positive cells (see black arrow). $B$, Section labeled with both GFAP and bFGF. Arrows point to three GFAP-IR astrocytes. The white arrow points to an astrocyte that was not labeled for bFGF. The black arrows point to two astrocytes that were labeled with both bFGF and GFAP. (Oil immersion: Scale bar, $25 \mu \mathrm{m})$.

directly into the VTA have been found to block the development of sensitization to amphetamine (Cador et al., 1997; JakeMatthews et al., 1997; Kim and Vezina, 1998). Finally, it is known that the development of sensitization to amphetamine can be blocked by systemic injections of protein synthesis inhibitors (Robinson, 1991; Karler et al., 1993) and that their direct application in the VTA, but not NAcc, can prevent sensitization to cocaine (Sorg and Ulibarri, 1995). This evidence, together with the finding that bFGF expression was increased in DA cell body regions but not in terminal regions, increases the probability that the enhanced astrocytic bFGF expression seen in the VTA and $\mathrm{SNc}$ regions is part of the cascade of intracellular and intercellular events that leads to long-lasting changes in the control and functioning of DA neurons. It should be pointed out, however, that bFGF-IR was assessed in striatal terminal regions only at 1 week after the last amphetamine injection. Thus, increases in bFGF-IR within these regions either at earlier or later times cannot be ruled out and are being investigated. This is particularly important in view of recent studies on drug-induced changes in postsynaptic neurons in the striatum (Moratalla et al., 1996; Nestler et al., 1996; Robinson and Kolb, 1997; Fienberg et al., 1998).

It can now be asked, through what mechanisms bFGF might play a role in the development of sensitization to the behavioral and neurochemical effects of amphetamine? It will be recalled that bFGF promotes growth and survival of embryonic DA neurons (Chadi et al., 1993; Bouvier and Mytilineou, 1995; Takayama et al., 1995; Hou et al., 1997) and this effect is mediated by astrocytes (Engele and Bohn, 1991). In adult animals, bFGF expression is increased after injury to midbrain DA neurons (Chadi et al., 1994) and appears to participate in the survival and sprouting of injured neurons (Chadi et al., 1993; Kawamata et al., 1997). Although amphetamine treatment has not been associated with the significant reductions of DA and metabolites in terminal regions seen after partial lesions within the DA system (Robinson and Whishaw, 1988), repeated administration of stimulant drugs can induce injury-like changes in the somatodendritic regions of midbrain DA neurons (Self and Nestler, 1995; Berhow et al.,
1996; Sklair-Tavron et al., 1996). The increased glutamatergic activity elicited by amphetamine and other stimulant drugs may, by placing excessive demands on the functioning of DA neurons, recruit regulatory and neuroprotective factors that stimulate recovery-like processes leading to enduring increases in DA neuron functioning and connectivity.

Interestingly, recent work from our laboratory suggests that the behavioral and neurochemical recovery seen 2-4 weeks after partial 6-OHDA lesions of nigrostriatal DA neurons (Robinson and Whishaw, 1988; Castañeda et al., 1990; Robinson et al., 1994) is facilitated by the actions of glutamate, presumably released as a result of neuronal stress and injury. We have found that recovery can be blocked by daily injections of NMDA antagonists given during the first week after injury (Emmi et al., 1996), making it appear that the compensatory changes in the remaining DA neurons are stimulated, at least in part, by glutamatergic activity. These findings suggest some similarity between the compensatory mechanisms seen after 6-OHDA lesions and those that underlie amphetamine-induced sensitization (Emmi et al., 1996, 1997). This idea is made more compelling by the fact that both after 6-OHDA lesions and after repeated exposure to amphetamine there is a sustained increase in astrocytic bFGF-IR in the SNc and VTA.

Another potential mechanism whereby bFGF could participate in the development of sensitization to the effects of amphetamine is by regulating the expression of glutamate receptor in DA neurons. It is known that bFGF increases the AMPA GluR1 subunit in cultured hippocampal neurons, and, as a consequence, the associated calcium response to AMPA receptor activation (Cheng et al., 1995). It is interesting, therefore, that increased expression of GluR1 subunit in DA somatodendritic regions (Fitzgerald et al., 1996) and increased sensitivity of DA neurons to AMPA activation (Zhang et al., 1997) have both been observed after repeated treatment with stimulant drugs. Notably, upregulation of the GluR1 subunit in the VTA has been shown to induce a sensitized-like response to the locomotor stimulant effects of morphine (Carlezon et al., 1997). In addition, both stimulant drugs and bFGF appear to increase GluR1 via post- 
transcriptional mechanisms (Mattson et al., 1993; Wolf, 1998). Thus, it is possible that bFGF participates in the development of DA sensitization by increasing GluR1 expression in DA neurons.

In summary, repeated exposure to amphetamine elicits marked increases in astrocytic bFGF-IR in midbrain DA somatodendritic regions that appear to be mediated by glutamate. Most importantly, these increases are long-lasting, continuing to be evident 1 month after the last exposure to amphetamine. These findings suggest that increased astrocytic expression of bFGF in DA somatodendritic regions is a mechanism by which stimulant drugs exert their long-term effects on midbrain DA function.

\section{REFERENCES}

Berhow M, Hiroi N, Kobierski L, Hyman S, Nestler E (1996) Influence of cocaine on the JAK-STAT pathway in the mesolimbic dopamine system. J Neurosci 16:8019-8026.

Bjijou Y, Stinus L, Le Moal M, Cador M (1996) Evidence for a selective involvement of dopamine D1 receptors of the ventral tegmental area in the behavioral sensitization induced by intra-ventral tegmental area injections of D-amphetamine. J Pharmacol Exp Ther 277:1177-1187.

Bouvier M, Mytilineou C (1995) Basic fibroblast growth factor increases division and delays differentiation of dopamine precursors in vitro. J Neurosci 15:7141-7149.

Cador M, Bjijou Y, Stinus L (1995) Evidence of a complete independence of the neurobiological substrates for the induction and expression of behavioral sensitization to amphetamine. Neuroscience 65:385-395.

Cador M, Bjijou Y, Cailhol S, Stinus L (1997) Role of a glutamatergic prefrontal/VTA innervation on amphetamine-induced behavioral sensitization to amphetamine. Soc Neurosci Abstr 23:1093.

Carlezon WJ, Boundy V, Haile C, Lane S, Kalb R, Neve R, Nestler E (1997) Sensitization to morphine induced by viral-mediated gene transfer. Science 277:812-814.

Castañeda E, Whishaw IQ, Robinson TE (1990) Changes in striatal dopamine neurotransmission assessed with microdialysis following recovery from a bilateral 6-OHDA lesion: variation as a function of lesion size. J Neurosci 10:1847-1854.

Chadi G, Møller A, Rosén L, Janson A, Agnati L, Goldstein M, Ögren S-O, Pettersson R, Fuxe K (1993) Protective actions of human recombinant basic fibroblast growth factor on MPTP-lesioned nigrostriatal dopamine neurons after intraventricular infusion. Exp Brain Res 97:145-158.

Chadi G, Cao Y, Pettersson RF, Fuxe K (1994) Temporal and spatial increase of astroglial basic fibroblast growth factor synthesis after 6-hydroxydopamine-induced degeneration of the nigrostriatal dopamine neurons. Neuroscience 61:891-910.

Cheng B, Furukawa K, O'Keefe J, Goodman Y, Kihiko M, Fabian T, Mattson M (1995) Basic fibroblast growth factor selectively increases AMPA-receptor subunit GluR1 protein level and differentially modulates $\mathrm{Ca} 2+$ responses to AMPA and NMDA in hippocampal neurons. J Neurochem 65:2525-2536.

Emmi A, Rajabi H, Stewart J (1996) Behavioral and neurochemical recovery from partial 6-hydroxydopamine lesions of the substantia nigra is blocked by daily treatment with glutamate receptor antagonists, MK-801 and CPP. J Neurosci 16:5216-5224.

Emmi A, Rajabi H, Stewart J (1997) Behavioral and neurochemical recovery from partial 6-hydroxydopamine lesions of the substantia nigra is blocked by daily treatment with $\mathrm{D} 1 / \mathrm{D} 5$, but not $\mathrm{D} 2$, dopamine receptor antagonists. J Neurosci 17:3840-3846.

Engele J, Bohn MC (1991) The neurotrophic effects of fibroblast growth factors on dopaminergic neurons in vitro are mediated by mesencephalic glia. J Neurosci 11:3070-3078.

Fienberg A, Hiroi N, Mermelstein P, Song W-J, Snyder G, Nishi A, Cheramy A, O'Callaghan J, Miller D, Cole D, Corbett R, Haile C, Cooper D, Onn S, Grace A, Ouimet C, White F, Hyman S, Surmeier D, Girault J-A, Nestler E, Greengard P (1998) DARPP-32: regulator of the efficacy of dopaminergic neurotransmission. Science 281:838-842.

Fitzgerald L, Ortiz J, Hamedani A, Nestler E (1996) Drugs of abuse and stress increase the expression of GluR1 and NMDAR1 glutamate receptor subunits in the rat ventral tegmental area: common adaptations among cross-sensitizing agents. J Neurosci 16:274-282.

Heidbreder C, Thompson A, Shippenberg T (1996) Role of extracellular dopamine in the initiation and long-term expression of behavioral sensitization to cocaine. J Pharmacol Exp Ther 278:490-502.
Hooks MS, Jones GH, Liem BJ, Justice JBJ (1992) Sensitization and individual differences to IP amphetamine, cocaine or caffeine following repeated intracranial amphetamine infusions. Pharmacol Biochem Behav 43:815-823.

Hou J-GG, Cohen G, Mytilineou C (1997) Basic fibroblast growth factor stimulation of glial cells protects dopamine neurons from 6-hydroxydopamine toxicity: involvement of the glutathione system. J Neurochem 69:76-83.

Hsu S, Raine L, Fanger H (1981) The use of anti-avidin antibody and avidin-biotin-peroxidase complex in immunoperoxidase techniques. Am J Clin Pathol 75:816-821.

Jake-Matthews C, Jolly D, Queen A, Brose J, Vezina P (1997) The competitive NMDA receptor antagonist CGS19755 blocks the development of sensitization of the locomotor and dopamine activating effects of amphetamine. Soc Neurosci Abstr 23:1092.

Kalivas PW, Alesdatter JE (1993) Involvement of NMDA receptor stimulation in the VTA and amygdala in behavioral sensitization to cocaine. J Pharmacol Exp Ther 267:486-495.

Kalivas PW, Duffy P (1993) Time course of extracellular dopamine and behavioral sensitization to cocaine I. Dopamine axon terminals. J Neurosci 13:266-275.

Kalivas PW, Duffy P (1995a) D1 receptors modulate glutamate transmission in the ventral tegmental area. J Neurosci 15:5379-5388.

Kalivas PW, Duffy P (1995b) Selective activation of dopamine transmission in the shell of the nucleus accumbens by stress. Brain Res 675:325-328.

Kalivas PW, Duffy P (1998) Repeated cocaine administration alters extracellular glutamate in the ventral tegmental area. J Neurochem 70:1497-1502.

Kalivas PW, Stewart J (1991) Dopamine transmission in the initiation and expression of drug- and stress-induced sensitization of motor activity. Brain Res Rev 16:223-244.

Kalivas PW, Weber B (1988) Amphetamine injection into the A10 dopamine region sensitizes rats to peripheral amphetamine and cocaine. J Pharmacol Exp Ther 245:1095-1102.

Karler R, Calder LD, Chaudhry IA, Turkanis SA (1989) Blockade of "reverse tolerance" to cocaine and amphetamine by MK-801. Life Sci 45:599-606.

Karler R, Chaudhry IA, Calder LD, Turkanis SA (1990) Amphetamine behavioral sensitization and the excitatory amino acids. Brain Res 537:76-82.

Karler R, Calder LD, Turkanis SA (1991) DNQX blockade of amphetamine behavioral sensitization. Brain Res 552:295-300.

Karler R, Calder LD, Thai LH, Bedingfield JB (1994) A dopaminergicglutamatergic basis for the action of amphetamine and cocaine. Brain Res 658:8-14.

Karler R, Finnegan K, Calder L (1993) Blockade of behavioral sensitization to cocaine and amphetamine by inhibitors of protein synthesis. Brain Res 603:19-24.

Kawamata T, Dietrich W, Schallert T, Gotts J, Cocke R, Benowitz L, Finklestein S (1997) Intracisternal basic fibroblast growth factor enhances functional recovery and up-regulates the expression of a molecular marker of neuronal sprouting following focal cerebral infarction. Proc Natl Acad Sci USA 94:8179-8184.

Kim J-H, Vezina P (1998) Metabotropic glutamate receptors are necessary for sensitization by amphetamine. NeuroReport 9:403-406.

Kolta MG, Shreve P, De Souza V, Uretsky NJ (1985) Time course of the development of the enhanced behavioral and biochemical responses to amphetamine after pretreatment with amphetamine. Neuropharmacology 24:823-829.

Kuczenski R, Segal D, Todd P (1997) Behavioral sensitization and extracellular dopamine responses to amphetamine after various treatments. Psychopharmacology 134:221-229.

Li Y, Vartanian A, White F, Xue C-J, Wolf M (1997) Effects of the AMPA receptor antagonist NBQX on the development and expression of behavioral sensitization to cocaine and amphetamine. Psychopharmacology 134:266-276.

Masserano J, Baker I, Natsukari N, Wyatt R (1996) Chronic cocaine administration increases tyrosine hydroxylase activity in the ventral tegmental area through glutaminergic- and dopaminergic D2-receptor mechanisms. Neurosci Lett 217:73-76.

Matsuzaki K, Yoshitake Y, Matuo Y, Sasaki H, Nishikawa K (1989) Monoclonal antibodies against heparin-binding growth factor II/basic fibroblast growth factor that block its biological activity: invalidity of 
the antibodies for tumor angiogenesis. Proc Natl Acad Sci USA 86:9911-9915.

Mattson MP, Kumar K, Cheng B, Wang H, Michaelis EK (1993) Basic FGF regulates the expression of a functional $71 \mathrm{kDa}$ NMDA receptor protein that mediates calcium influx and neurotoxicity in cultured hippocampal neurons. J Neurosci 13:4575-4588.

Moratalla R, Elibol B, Vallejo M, Graybiel A (1996) Network-level changes in expression of inducible Fos-Jun proteins in the striatum during chronic cocaine treatment and withdrawal. Neuron 17:147-156.

Nestler E, Berhow M, Brodkin E (1996) Molecular mechanisms of drug addiction: adaptations in signal transduction pathways. Mol Psychiatry 1:190-199.

Paulson PE, Robinson TE (1995) Amphetamine-induced timedependent sensitization of dopamine neurotransmission in the dorsal and ventral striatum: a microdialysis study in behaving rats. Synapse 19:56-65.

Paulson PE, Camp DM, Robinson TE (1991) Time course of transient behavioral depression and persistent behavioral sensitization in relation to regional brain monoamine concentrations during amphetamine withdrawal in rats. Psychopharmacology 103:480-492.

Paxinos G, Watson C (1997) The rat brain in stereotaxic coordinates. New York: Academic.

Pechan PA, Chowdhury K, Gerdes W, Seifert W (1993) Glutamate induces the growth factors NGF, bFGF, the receptor FGF-R1 and c-fos mRNA expression in rat astrocyte culture. Neurosci Lett 153:111-114.

Perugini M, Vezina P (1994) Amphetamine administered to the ventral tegmental area sensitizes rats to the locomotor effects of nucleus accumbens amphetamine. J Pharmacol Exp Ther 270:690-696.

Robinson TE (1991) The neurobiology of amphetamine psychosis: evidence from studies with an animal model. In: Biological basis of schizophrenia (Nakazawa T, ed) pp 185-201. Tokyo: Japan Scientific Societies.

Robinson TE, Becker JB (1986) Enduring changes in brain and behavior produced by chronic amphetamine administration: a review and evaluation of animal models of amphetamine psychosis. Brain Res Rev 396:157-198.

Robinson TE, Whishaw IQ (1988) Normalization of extracellular dopamine in striatum following recovery from a partial unilateral 6-OHDA lesion of the substantia nigra: a microdialysis study in freely moving rats. Brain Res 450:209-224.

Robinson TE, Kolb B (1997) Persistent structural modifications in nucleus accumbens and prefrontal cortex neurons produced by previous experience with amphetamine. J Neurosci 17:8491-8497.

Robinson TE, Jurson PA, Bennett JA, Bentgen KM (1988) Persistent sensitization of dopamine neurotransmission in ventral striatum nucleus accumbens produced by prior experience with +-amphetamine: a microdialysis study in freely moving rats. Brain Res 462:211-222.

Robinson TE, Mocsary Z, Camp DM, Whishaw IQ (1994) Time course of recovery of extracellular dopamine following partial damage to the nigrostriatal dopamine system. J Neurosci 14:2687-2696.

Self DW, Nestler EJ (1995) Molecular mechanisms of drug reinforcement and addiction. Annu Rev Neurosci 18:463-495.

Sklair-Tavron L, Shi W-X, Lane S, Harris H, Bunney B, Nestler E (1996) Chronic morphine induces visible changes in the morphology of mesolimbic dopamine neurons. Proc Natl Acad Sci USA 93:11202-11207.

Sorg BA, Ulibarri C (1995) Application of a protein synthesis inhibitor into the ventral tegmental area, but not into the nucleus accumbens, prevents behavioral sensitization to cocaine. Synapse 20:217-224.
Stewart J, Druhan JP (1993) Development of both conditioning and sensitization of the behavioral activating effects of amphetamine is blocked by the non-competitive NMDA receptor antagonist, MK-801. Psychopharmacology 110:125-132.

Stone TW (1993) Neuropharmacology of quinolinic and kynurenic acids. Pharmacol Rev 45:309-379.

Szele F, Alexander C, Chesselet M-F (1995) Expression of molecules associated with neuronal plasticity in the striatum after aspiration and thermocoagulatory lesions of the cerebral cortex in adult rats. J Neurosci 15:4429-4448.

Taber MT, Fibiger HC (1995) Electrical stimulation of the prefrontal cortex increases dopamine release in the nucleus accumbens of the rat: modulation by metabotropic glutamate receptors. J Neurosci 15:3896-3904.

Takayama H, Ray J, Raymon H, Baird A, Hogg J, Fisher L, Gage F (1995) Basic fibroblast growth factor increases dopaminergic graft survival and function in a rat model of Parkinson's disease. Nature Med 1:53-58.

Vezina P (1993) Amphetamine injected into the ventral tegmental area sensitizes the nucleus accumbens dopaminergic response to systemic amphetamine: an in vivo microdialysis study in the rat. Brain Res 605:332-337.

Vezina P, Stewart J (1990) Amphetamine administered to the ventral tegmental area but not to the nucleus accumbens sensitizes rats to systemic morphine: lack of conditioned effects. Brain Res 516:99-106.

White FJ, Hu XT, Zhang XF, Wolf ME (1995) Repeated administration of cocaine or amphetamine alters neuronal responses to glutamate in the mesoaccumbens dopamine system. J Pharmacol Exp Ther 273:445-454.

Wolf M (1998) The role of excitatory amino acids in behavioral sensitization to psychomotor stimulants. Prog Neurobiol 54:679-720.

Wolf ME, Jeziorski M (1993) Coadministration of MK-801 with amphetamine, cocaine or morphine prevents rather than transiently masks the development of behavioral sensitization. Brain Res 613:291-294.

Wolf ME, Khansa MR (1991) Repeated administration of MK-801 produces sensitization to its own locomotor stimulant effects but blocks sensitization to amphetamine. Brain Res 562:164-168.

Wolf M, Xue C (1998) Amphetamine and D1 dopamine receptor agonists produce biphasic effects on glutamate efflux in rat ventral tegmental area: modification by repeated amphetamine administration. J Neurochem 70:198-209.

Wolf ME, White FJ, Nassar RN, Brooderson RJ, Khansa MR (1993) Differential development of autoreceptor supersensitivity and enhanced dopamine release during amphetamine sensitization. J Pharmacol Exp Ther 264:249-255.

Wolf ME, White FJ, Hu X-T (1994) MK-801 prevents alterations in the mesoaccumbens dopamine system associated with behavioral sensitization to amphetamine. J Neurosci 14:1735-1745.

Xue C, Ng J, Li Y, Wolf M (1996) Acute and repeated systemic amphetamine administration: effects on extracellular glutamate, aspartate, and serine levels in rat ventral tegmental area and nucleus accumbens. J Neurochem 67:352-363.

Zhang X, Hu X, White F, Wolf M (1997) Increased responsiveness of ventral tegmental area dopamine neurons to glutamate after repeated administration of cocaine or amphetamine is transient and selectively involves AMPA receptors. J Pharmacol Exp Ther 281:699-706. 